\title{
Treatment of severe mitral regurgitation caused by lesions in both leaflets using multiple mitral valve plasty techniques in a small dog
}

\author{
Satoko Yokoyama ${ }^{1,2}$, Isamu Kanemoto ${ }^{1, *}$, Kippei Mihara ${ }^{1}$, Takanori Ando ${ }^{1,3}$, Koudai Kawase ${ }^{1,4}$, Yasuaki Sahashi ${ }^{1,5}$ \\ and Kazuhito Iguchi ${ }^{1,6}$ \\ ${ }^{1}$ Chayagasaka Animal Hospital, 1-1-5 Shinnishi, Chikusa, Nagoya, Aichi, 464-0003 Japan \\ ${ }^{2}$ Miyashita Animal Hospital, 5-8-29 Kegoya, Kure, Hiroshima, 737-0012 Japan \\ ${ }^{3}$ Heart-Will Animal Hospital, 1-2-14 Okidai, Tobata, Kita-Kyushu, Fukuoka, 804-0064 Japan \\ ${ }^{4}$ Sapporo Night Animal Hospital Emergency \& Critical Care, 7-5-6 Minami-2 Zyou-nishi, Chuo, Sapporo, \\ Hokkaido, 060-0062 Japan \\ ${ }^{5}$ Garcia Animal Hospital, 2-1-56 Tatumi, Koutou-ku, Tokyo, 135-0053 Japan \\ ${ }^{6}$ Momijiyama Douri Pet Clinic, 5-2-9 Nakano, Nakano-ku, Tokyo, 164-0001 Japan
}

\begin{abstract}
Mitral valve plasty (MVP) is preferred over mitral valve replacement (MVR) for mitral regurgitation in humans because of its favorable effect on quality of life. In small dogs, it is difficult to repair multiple lesions in both leaflets using MVP. Herein, we report a case of severe mitral regurgitation caused by multiple severe lesions in the posterior leaflet (PL) in a mixed Chihuahua. Initially, we had planned MVR with an artificial valve. However, MVP combined with artificial chordal reconstruction of both leaflets, semicircular suture annuloplasty, and valvuloplasty using a newly devised direct scallop suture for the PL was attempted in this dog. The dog recovered well and showed no adverse cardiac signs, surviving two major operations. The dog died 4 years and 10 months after the MVP due to noncardiovascular disease. Our additional technique of using a direct scallop suture seemed useful for PL repair involving multiple scallops in a small dog.
\end{abstract}

Keywords: Chordal reconstruction, Dog, Mitral regurgitation, Multiple direct scallop suture, Semi-circular suture annuloplasty.

\section{Introduction}

Mitral regurgitation (MR) is the most common cardiac disease in old, small dogs (Kittleson and Kiene, 1998). Mitral valve plasty (MVP) is the preferred surgical treatment over mitral valve replacement (MVR) for MR in humans because of its positive effect on quality of life (Carpentier et al., 2010). Recently, there have been increased reports of using MVP to treat MR in dogs (Kanemoto et al., 1990, 2004, 2017; Boggs et al., 1996; Griffiths et al., 2004; Uechi, 2012).

However, there have been few reports of the application of MVP to treat severe MR involving the posterior leaflet (PL) in small dogs (Boggs et al., 1996; Uechi, 2012; Kanemoto et al., 2017), in whom MVR was previously indicated (Orton et al., 2005; Taguchi et al., 2014).

Herein, we describe a case of severe MR caused by multiple lesions in the PL in a mixed Chihuahua, who was treated with a combination of the following three MVP techniques: artificial chordal reconstruction (CR) for the anterior leaflet (AL) and the PL, semicircular suture annuloplasty (AP) for a dilated mitral annulus, and valvuloplasty (VP) using newly devised direct scallop sutures for the PL.

\section{Case Details}

The patient was a male mixed Chihuahua (age, 10 years and 2 months; weight, $6.1 \mathrm{~kg}$; body condition score [BCS], IV/V). At the time of our initial examination, the dog had a history of arrhythmia since a young age and had been diagnosed with MR one year ago. Medical treatment was started by a family doctor in Tokyo, Japan. The dog also had a fainting episode several days before visiting our hospital.

The day before, the dog presented for abrupt shock at a night-emergency hospital, and was diagnosed with cardiac effusion. The dog had a hard cough during rest despite being given pimobendan $(0.42 \mathrm{mg} / \mathrm{kg})$, enalapril $(0.42 \mathrm{mg} / \mathrm{kg})$, furosemide $(1.7 \mathrm{mg} / \mathrm{kg})$, and spironolactone $(2.1 \mathrm{mg} / \mathrm{kg})$ twice daily via perioral administration.

\section{Examination findings}

Auscultation revealed a grade $3 / 6$ systolic murmur at the apex of the heart, and rough vesicular sounds in the lung.

Thoracic radiography revealed severe left heart enlargement (vertebral heart scale [VHS]: 12.8 vertebra and cardio-thoracic ratio [CTR]: $80.1 \%$ ) and increased pulmonic vascular shading (Fig. 1A, Table 1). 
Table 1. Pre- and post-operative echocardiography and thoracic radiography findings.

\begin{tabular}{lcccccc}
\hline & \multirow{2}{*}{ Pre-op } & Post-op & Post-op & Post-op Post-op & Post-op \\
& $3 \mathrm{~m}$ & $1 \mathrm{y}$ & $2 \mathrm{y}$ & $3 \mathrm{y}$ & $4 \mathrm{y} 3 \mathrm{~m}$ \\
\hline MR & severe & slight & slight & mild & mild & mild \\
TR & slight & mild & mild & mild & mild & moderate \\
LVIDd & 40.9 & 27 & 27 & 23 & 23 & 24 \\
LA/Ao & 3.5 & 1.62 & 1.62 & 1.33 & 1.29 & 1.43 \\
VHS & 12.8 & 10.7 & 10.6 & 10 & 11 & 11.3 \\
\hline
\end{tabular}

(Pre-op): preoperative; (Post-op): postoperative; (m): month; (y): year; (MR): mitral regurgitation (semi-quantitative method); (TR): tricuspid regurgitation (the same as MR); (LVIDd): left ventricular end-diastolic internal dimension: mm, (LA/Ao): left atrium/aorta ratio; (VHS): vertebral heart scale.
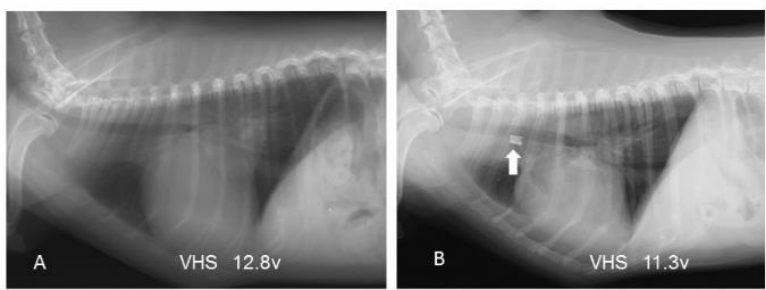

Fig. 1. Pre- and post-operative thoracic radiograms. (A): Preoperative X-ray photograph reveals severe left cardiac enlargement (VHS 12.8 v). (B): Post-operative X-ray photograph at 4 years and 3 months reveals mild right cardiac enlargement (VHS $11.3 \mathrm{v}$ ). An arrow of anterior thorax in B photograph shows the hemoclips of right lung lobectomy due to lung cancer at 3 years 5 months post-operation. (VHS): vertebral-heart scale.

Color-Doppler echocardiography revealed severe MR and slight tricuspid regurgitation (TR) (Fig. 2A, Table 1). Imaging in B-mode showed no cardiac effusion and several elongated or ruptured chordae in the AL and PL (Fig. 2A), while M-mode showed significant dilatation of the left heart (left ventricular end-diastolic internal dimension [LVIDd]: $40.9 \mathrm{~mm}$ and left atrium/aorta ratio [LA/Ao]: 3.5) (Table 1, LVIDd, LA/Ao).

Based on the results of these examinations, he was diagnosed with severe MR (ACVIM stage D; Atkins et al., 2009) due to multiple elongated or ruptured chordae and mild TR. Mitral valve plasty was planned in accordance with the owner's strong wishes, although hard cough becomes mild with additional injection of flosemide.

\section{Surgical methods}

We had planned to perform MVR with a bioprosthetic valve in this case, if it was not possible to perform MVP. We began the surgical procedures with surfaceinduced hypothermia combined with cardiopulmonary bypass (CPB) (Kanemoto et al., 2010, Mihara et al., 2017).

After inducing anesthesia, a venous cannula and an arterial cannula were inserted into the left jugular vein and the left carotid artery, respectively, and both connected to the CPB circuit (Terumo Baby ${ }^{\circledR}, \mathrm{RX}-05$ : Terumo Baby; Terumo Co., Tokyo, Japan).

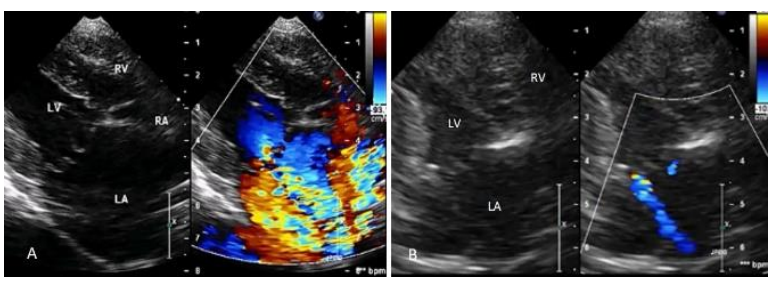

Fig. 2. Pre- and post-operative B- and color-mode echocardiogram. (A): Pre-operative color-Doppler echocardiogram (right side) shows severe mitral regurgitation (MR) and slight tricuspid regurgitation (TR). The B-mode (left side) image shows several elongated or ruptured chordae in anterior and posterior leaflets. (B): Post-operative colorDoppler (right side) reveals small MR at 4 years 3 months after operation. (LA): left atrium; (LV): left ventricle; (RA): right atrium; (RV): right ventricle.

After aortic cross clamp and cardioplegia, we opened the left atrium via $5^{\text {th }}$ left thoracotomy, and assessed the damaged mitral valve. We found that two strut chordae (A1-2 and A2-3) of the AL were elongated and 4 chordae (P1, P1-2, P2-3, P3) of the PL were ruptured. The margins of both leaflets were hypertrophied and deep gaps in the PL were noted (Fig. 3A). Therefore, we planned to use three MVP techniques in this surgery: artificial chordal reconstruction (CR), semicircular suture annuloplasty (AP), and valvuloplasty (VP). First, we performed CR using a pair of e-PTFE sutures, each manipulated with two needles (Gore-Tex®, CV-6: Gore-Tex; WL Gore \& Associates, Inc., Flagstaff, Arizona, USA), which were previously prepared by triple-tying in the center and attaching to a pledget (Fig. 3C). This suture pair was sutured with a mattress and fixed as a sandwich-shape with another pledget at the anterior papillary muscle (Fig. 3B). Next, one pair of sutures was used to suture both sides of the A1-2 chorda of the AL, followed by adjusting the length to the position of mitral ring and ligation. The other pair of sutures was used to suture both the P1 and P1-2 chordae of the PL, followed by ligation (Fig. 3B, red lines).

In the same manner, the second pair of e-PTFE sutures was used at the posterior papillary muscle and one pair of sutures was used to suture both sides of the A2-3 chorda of the AL, followed by ligation. Another pair of sutures was used to suture both the P2 and P3 chordae of the PL, followed by ligation (Fig. 3B, red lines). For VP, the deep gaps between the P1 and P2 scallops, and between the P2 and P3 scallops, of the PL were sutured with a direct cross suture using a polypropylene suture (Proline ${ }^{\circledR}$ 6-0: Prolene; Ethicon, Somerville, New Jersey, USA) (Fig. 3B, blue lines). Finally, semicircular suture AP using a Proline 4-0 suture attached to a pledget with two needles was performed by alternately suturing from the anterior base to the posterior base of the AL, through the fibrous ring of the PL, and attaching to another pledget, followed by ligation. 

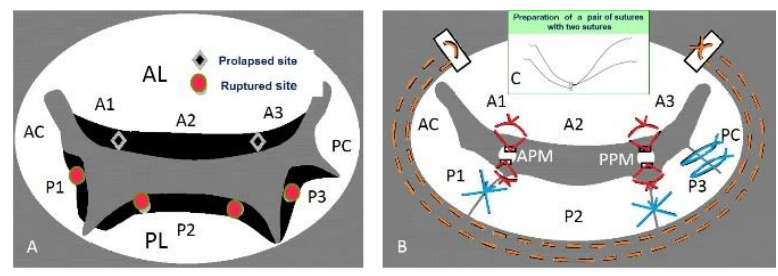

Fig. 3. Mitral valve lesion site in mitral valve map and mitral valve plasty diagram. (A): $\mathrm{A} 1, \mathrm{~A} 2$, and $\mathrm{A} 3$ in $\mathrm{AL}$ contact to $\mathrm{P} 1, \mathrm{P} 2$, and $\mathrm{P} 3$ in PL as shown in the mitral valve map. Black diamond shapes show two prolapsed chordae due to elongation in A1-2 and A2-3 in AL. Red round shapes show four ruptured chordae in P1, P2, and P3 in PL. Black margin of $\mathrm{AL}$ and PL are remarkably hypertrophied. (AL): anterior leaflet; (PL): posterior leaflet; $(\mathrm{AC})$ : anterior commissure; (PC): posterior commissure. (B): Red lines show 4 chordal reconstructions in A1-2, A2-3 chordae in AL, and P1-P2, P2P3 chordae in PL. Blue lines show three direct scallop sutures in P1-P2, P2-P3 scallops, and P3-PC scallop and leaflet in PL. Brown dotted lines show semicircular suture annuloplasty with continuous suturing from the anterior to the posterior base in AL through the fibrous ring of PL. (C) (at top middle of Fig. B): Two ePTFE sutures with triple-tying in the center and attached to a pledget are previously prepared. (APM): anterior papillary muscle; (PPM): posterior papillary muscle.

This was performed to diminish the diameter of the mitral valve ring from $20 \mathrm{~mm}$ (preoperative) to $13 \mathrm{~mm}$ in size (the same size as the AL), as measured using a sizer (Fig. 3B, brown lines). As the result of a backflow test, the gap between the P3 and posterior commissure (PC) scallops was additionally sutured with two direct-knotted sutures using the same suture (Fig. 3B, blue lines). As a result, MR flow mostly disappeared. The left atrium and chest were closed in a routine manner.

\section{Postoperative course}

Although the dog's immediate postoperative course was good, the dog remained in hospital for 21 days until discharge because of a wound infection. Three months after the operation, thoracic radiography revealed that the VHS had decreased from $12.8 \mathrm{v}$ before operation to $10.7 \mathrm{v}, 10.6 \mathrm{v}, 10.0 \mathrm{v}, 11.0 \mathrm{v}$, and $11.3 \mathrm{v}$ at 3 months, 1 year, 2 years, 3 years, and 4 years 3 months postoperatively, respectively. (Fig. 1A, 1B, Table 1). Color-Doppler echocardiography revealed slight MR and mild TR at 3 months and 1 year, mild MR and TR at 2 years and 3 years, and mild MR and moderate TR at 4 years 3 months after the operation (Fig. 2B, Table 1). M-mode images revealed that LVIDd and LA/Ao were remarkably reduced from $40.9 \mathrm{~mm}$ and 3.5 before the operation to $27.0 \mathrm{~mm}$ and 1.62 at 3 months and 1 year, $23 \mathrm{~mm}$ and 1.33 at 2 years, $23 \mathrm{~mm}$ and 1.29 at 3 years, and $24 \mathrm{~mm}$ and 1.43 at 4 years 3 months postoperation, respectively (Table 1, LVIDd, LA/Ao). The dog safely underwent two major operations: a splenectomy due to a splenic tumor at 9 months postMVP and a right-lung lobectomy due to lung cancer at
3 years 5 months post-MVP. However, the dog abruptly died from acute pancreatitis and an epileptic attack (the latter was an illness that was identified preoperatively) in the hospital of a family doctor. After MVP, he experienced no cardiac events. Since 2 months postMVP, the dog received only enalapril until death at his family hospital. Unfortunately, the owner did not allow us to perform an autopsy.

\section{Discussion}

There are three main techniques of MVP: (1) CR for ruptured or elongated chordae tendineae, (2) AP for a dilated mitral annulus to increase the coaptation area of the AL and PL, and (3) VP for deformed or redundant valve leaflets (Carpentier et al., 1971).

To treat severe MR caused by ruptured chordae of the AL, Kanemoto et al. (2017) reported the use of a basic MVP technique that combined CR using ePTFE sutures for the $\mathrm{AL}$ and semicircular suture $\mathrm{AP}$ using polypropylene sutures for the dilated mitral annulus. However, in a severe case of MR complicated by the presence of multiple lesions in both leaflets, two additional techniques are required: valve scallop suture VP for the PL and anterior commissure AP (the Kay method) (Kay and Egerton, 1963). In the present case, two additional techniques of CR and VP for the PL were also required, in addition to the basic MVP technique. Specifically, two CR procedures were used at both ends of the P1 and P2 scallops, and P2 and P3 scallops in the PL. In addition, both gaps of the P1 and $\mathrm{P} 2$ scallops, and P2 and P3 scallops were sutured in a direct-cross fashion. Additionally, a gap at the P3 and PC scallops was sutured using two single, direct sutures. As a result, the MVP for the PL using CR with two artificial chordae, and VP with multiple direct scallop sutures of three gaps between the P1 and P2, P2 and P3, and P3 and PC scallops, transformed four scallops into one leaflet for the PL with no gap in between. In humans, MVP for a PL that is faulty due to a redundant valve is mainly used via rectangular dissection (McGoon, 1960) or triangular resection (Suri and Orsulak, 2005), although scallop sutures (known as a "magic suture") are used as an accessorial technique (Carpentier et al., 2010). In our case, the MVP used for the extensively damaged PL was basically a combination of $\mathrm{CR}$ for the ruptured or prolonged chordae and VP using multiple scallops that were directly sutured without resection; with this method, a reduction in the coaptation area of the AL and PL was avoided. The dog safely underwent two major operations - a splenectomy for a splenic tumor at 9 months and a right lung lobectomy for lung cancer at 3 years and 5 months post-MVP. Furthermore, he did not exhibit any signs of cardiac issues post-MVP. This case study reports, for the first time, new revised techniques of MVP for treating a severe lesion of the PL of the mitral valve in a small dog. Our additional technique of 
using a direct scallop suture seemed useful for PL repair involving multiple scallops in a small dog. We believe that our study makes a significant contribution to the literature because of its novelty in veterinary medicine.

\section{Acknowledgments}

This paper was presented, in part, at the $92^{\text {nd }}$ Japanese Society of Veterinary Cardiology meeting in Ohmia, Japan, on July 31-August 1, 2010. We would like to thank Editage (www.editage.jp) for English language editing.

\section{Conflict of interest}

The authors declare that there is no conflict of interest.

\section{References}

Atkins, C., Bonagura, J., Ettinger, S., Fox, P., Gordon, S., Haggstrom, J., Hamlin, R., Keene, B., LuisFuentes, V. and Stepien, R. 2009. Guidelines for the diagnosis and treatment of canine chronic valvular heart disease. J. Vet. Inter. Med. 23, 1142-1150.

Boggs, L.S., Dewan, S.J. and Ballard, S.E. 1996. Mitral valve reconstruction in a toy-breed dog. J. Amer. Vet. Med. Assoc. 209, 1872-1876.

Carpentier, A., Adams, D.H. and Filsoufi, F. 2010. Techniques in type II: posterior leaflet prolapse. In Reconstructive valve surgery. Maryland Heights, MO: Elsevier, pp: 115-126.

Carpentier, A., Deloche, A., Dauptain, J., Soyer, R., Blondeau, P., Piwnica, A., Dubost, C. and McGoon D.C. 1971. A new reconstructive operation for correction of mitral and tricuspid insufficiency. J. Thorac. Cardiovasc. Surg. 61, 1-13.

Griffiths, L.G., Orton, E.C. and Boon, J.A. 2004. Evaluation of techniques and outcomes of mitral valve repair in dogs. J. Amer. Vet. Med. 224, 19411945.

Kanemoto, I., Shibata, S., Noguchi, H., Chimura, S., Kobayashi, M. and Shimizu, Y. 1990. Successful mitral valvuloplasty for mitral regurgitation in a dog. Jap. J. Vet. Sci. 52, 411-414.

Kanemoto, I., Suzuki, H., Taguchi, D., Yokoyama, S., Mizuno, M. and Kanamoto, T. 2004. Successful surgical repair for severe mitral regurgitation in five small-breed dogs. Vet. Surg. 33, 435-E12.
Kanemoto, I., Taguchi, D., Mihara, K., Kawase, K., Sato, K., Iwata, K. and Iwanaga, K. 2017. Longterm survival of two dogs after mitral valve plasty. Vet. Sci. Devel. 7, 51 (doi: 10.4081/vsd.2017.6335).

Kanemoto, I., Taguchi, D., Yokoyama, S., Mizuno, M., Suzuki, H. and Kanamoto T. 2010. Open heart surgery with deep hypothermia and cardiopulmonary bypass in small and toy dogs. Vet. Surg. 39, 674-679.

Kay, J.H. and Egerton, W.S. 1963. The repair of mitral insufficiency associated with ruptured chordae tendineae. Ann. Surg. 157, 351-360.

Kittleson, M.D. and Kiene, R.D. 1998. Myxomatous atrioventricular valvular degeneration. In Small Animal Cardiovascular Medicine, Eds., Kittleson, M.D. and Kiene, R.D., Kimberton, PA: Mosby, pp: 297-318.

McGoon, D.C. 1960. Repair of mitral insufficiency due to ruptured chordae tenedineae. J. Thorac. Cardiovasc. Surg. 39, 357-362.

Mihara, K., Kanemoto, I., Sato, K., Mori, T., Abe, H., Niimi, S., Yamada, K., Ohira, K., Andow, T., and Hirakawa, A. 2017. Open heart surgery with deep hypothermic cardiopulmonary bypass and more than 90 minutes of aortic cross clamp time in 10 small dogs. Vet. Sci. Devel. 7, 6442 (doi: 10.4081/vsd.2017.6442).

Orton, E.C., Hackett, T.B., Mama, K. and Boon, J.A. 2005. Technique and outcome of mitral valve replacement in dogs. J. Amer. Vet. Med. 226, 15081511.

Suri, R.M. and Orsulak, T.A. 2005. Triangular resection for repair of mitral regurgitation due to degenerative disease. Ope. Tech. Thorac. Cardiovasc. Surg. 10, 194-199.

Taguchi, D., Kanemoto, I., Yokoyama, S., Mizuno, M. and Washizu, M. 2014. Mitral valve replacement with a mechanical valve for severe mitral regurgitation in a small dog. Case Report Vet. Med. ID 892625(doi: org/10.1155/2014/892625).

Uechi, M. 2012. Mitral valve repair in dogs. J. Vet. Cardio. 14, 185-192. 